\section{La formación en extensión como desafío. Reflexiones a partir de la determinación curricular de la extensión agropecuaria en La Plata en los años 60}

\section{Cintia Analía Barrionuevo}

Becaria de investigación y doctoranda de la Facultad de Ciencias Agrarias y Forestales. Universidad Nacional de La Plata, Argentina. cintia.barrionuevo@agro.unlp.edu.ar
Juan José Garat

Docente investigador y Secretario de Extensión de la Facultad de Ciencias Agrarias y Forestales. Universidad Nacional de La Plata, Argentina. lolo_garat@yahoo.com.ar

\section{Resumen}

Según Barrientos (2002), la primera cátedra de "extensión agropecuaria" en Argentina se creó en la Universidad Nacional de La Plata en la segunda mitad de los años 60 . A casi 50 años de este proceso de determinación curricular, este artículo pretende contribuir no solo a una sociohistoria institucional y disciplinar en particular — que involucra a la entonces denominada Facultad de que, mediante la revisión bibliográfica y el análisis de documentos, compromete una experiencia que, influida por las teorías de la modernización y el enfoque difusionista, integraba las funciones de docencia e investigación y acercaba a los estudiantes de grado de la carrera de Ingeniería Agronómica a la práctica profesional extensionista. Consideramos que una reflexión crítica de este proceso puede abonar al debate de la formación en extensión universitaria. Buscando superar la dicotomía intramuros/extramuros con que nació la extensión, elementos para poner en discusión los sentidos de las intervenciones (más allá de lo rural/agropecuario), a la vez que permite reconocer tensiones aún vigentes. Agronomía de La Plata y al Instituto Nacional de Tecnología Agropecuaria- sino examinar la compleja génesis de un espacio político-pedagógico como éste suma
Integración de la docencia y la extensión /

Perspectivas

RECEPCIÓN: 24/06/16

ACEPTACIÓN FINAL: 10/10/16

\section{Resumo}

Segundo Barrientos (2002), a primeira cadeira de "extensão agropecuária" na Argentina foi criada na Universidade Nacional de La Plata, na segunda metade dos anos 60. Quase 50 anos depois deste processo de determinação curricular, este artigo tenta não procura apenas contribuir a uma sócio-história institucional e disciplinar particular — que envolve a então denominada Faculdade de Agronomia de La Plata e ao Instituto Nacional de Tecnologia Agropecuria- mas também, e através da revisão bibliográfica e a análise de documentos, engaja em uma experiência que, influenciada pelas teorias da modernização e da abordagem difusionista, integrava as funções de ensino e pesquisa e aproximava os alunos de graduação do curso de Engenharia Agronômica da prática profissional de extensão. Acreditamos que uma reflexão crítica deste processo possa ajudar a debater a formação em extensão universitária. Procurando superar a dicotomia intramuros/ extramuros com que nasceu a extensão, estudar a complexa gênese de um espaço político-pedagógico como este, adiciona elementos para colocar em discussão tanto os sentidos das intervenções (além do rural / agropecuário) quanto reconhecer tensões ainda em vigor.

\section{Palabras clave}

- Extensión rural

- Currícula de grado

- Formación de extensionistas

- Universidad Nacional de La Plata

- Instituto Nacional de Tecnología Agropecuaria
Palavras-chave

- Extensão rural

- Currículo de gradução

- Formação de extensão

- Instituto Nacional de Tecnologia Agropecuária
- Universidade Nacional de La Plata

\section{Para citación de este artículo}

Barrionuevo, A. C. y Garat, J. J. (2016). La formación en extensión como desafío. Reflexiones a partir de la determinación curricular de la extensión agropecuaria en La Plata en los años 60. En Revista +E versión digital, (6), pp. 64-73. Santa Fe, Argentina: Ediciones UNL. 


\title{
66
}

\author{
el programa traía al aula la experiencia de \\ técnicos de las agencias de extensión de otra \\ institución del complejo científico-tecnológico \\ nacional (INTA) y proponía que los estudiantes \\ avanzados de una carrera de grado lleven a \\ cabo prácticas en terreno
}

\begin{abstract}
"Sin duda no hay instrumento de ruptura más poderoso que la reconstrucción de la génesis; al hacer resurgir los conflictos y las confrontaciones de los primeros comienzos y, al mismo tiempo, los posibles descartes, reactualiza la posibilidad de que hubiera sido (y de que sea) de otra manera y, a través de esta utopía práctica, cuestiona lo posible que, entre todos los otros, se encuentra realizado." Pierre Bourdieu, Espíritus de Estado.
\end{abstract}

\section{Introducción}

Este artículo surge a partir del trabajo de archivo realizado por los autores para encontrar los antecedentes del actualmente denominado Curso de Extensión Rural en la Facultad de Ciencias Agrarias y Forestales (FCAyF) de la Universidad Nacional de La Plata (UNLP), institución académica a la cual Barrientos (2002) atribuye la creación de la primera cátedra de esta disciplina en Argentina. Actualmente, Extensión Rural ${ }^{1}$ es una asignatura de grado de 60 horas con una matrícula aproximada de 100 estudiantes, obligatoria para el último año de las carreras de Ingeniería Agronómica e Ingeniería Forestal y eventual optativa para estudiantes de otras facultades como Ciencias Veterinarias o Humanidades y Ciencias de la Educación. Desde este espacio se han articulado múltiples proyectos de docencia, investigación y extensión que entrelazaron de modo significativo diversos actores sociales de la región ampliada del Gran La Plata. En los últimos 15 años su vinculación con el medio rural viene siendo encuadrada en el enfoque dialógico-crítico de extensión (Freire, 1973), con herramientas teórico-conceptuales del desarrollo territorial rural, el enfoque interdisciplinario de los sistemas agroalimentarios localizados (SIAL), entre otras perspectivas, dentro de un marco programático de Educación/Comunicación. Antes del año 2000 era una asignatura optativa de 120 horas y un promedio aproximado de 10 alumnos.
El objeto de esta indagación es la reconstrucción del marco conceptual y las actividades contenidas en un Programa de Capacitación en Extensión Agropecuaria que data de 1966/1967 y que involucró al Instituto Nacional de Tecnología Agropecuaria (INTA) y a la, entonces denominada, Facultad de Agronomía de La Plata (FALP, hoy FCAyF). Este programa dio origen a la Cátedra de Extensión Agropecuaria en el plan de estudios de la carrera de ingeniería agronómica en 1969.

La principal fuente analizada, disparadora de muchas preguntas y pistas, fue el expediente del convenio suscripto entre la FALP e INTA. Sus fojas comienzan con una resolución de noviembre de 1966 firmada por el decano de la FALP mediante la cual resolvía crear un Programa de Capacitación en Extensión Agropecuaria en colaboración con INTA. Finalizando el expediente, en una nota de noviembre de 1975, el mencionado convenio era denunciado ya que "dado el tiempo transcurrido, este decanato estima que la Cátedra de Extensión Agropecuaria cuenta con personal docente lo suficientemente idóneo para tomar responsabilidad total de la misma". (UNLP, Expte 200-24194/67:122)

Entre los años 1966 y 1975 se dio un período de la historia argentina que requiere ser ponderado para comprender el contexto de creación de una cátedra de Extensión Agropecuaria en el '69 platense.

En primer lugar, 1966 es el año de inicio de la dictadura cívico militar autodenominada Revolución Argentina, bajo la presidencia de facto del general Juan Carlos Onganía. Es importante tener en cuenta que nos situamos en la segunda etapa del modelo de Industrialización por Sustitución de Importaciones (Campos, 2010); en el intento de implantar el Estado Burocrático Autoritario (O’Donnell, 1977) en la Argentina; en una dinámica políticosocial de alta conflictividad que tiene en el Cordobazo de 1969 
y otros "azos" sus hitos más reconocidos; y en el despliegue de políticas para el desarrollo de un modelo económico que, según los analistas, se venía agotando desde mediados de los años 50. ${ }^{2}$ Además, según Cavarozzi (1983) desde el '66 en adelante la modalidad de intervención de las fuerzas armadas sobre la política nacional se vuelve más violenta y autoritaria, en consonancia con la política exterior de Estados Unidos en los países latinoamericanos, a partir de la Doctrina de Seguridad Nacional en el contexto de la Guerra Fría.

En segundo lugar, la determinación (y estructuración) curricular que investigamos está emparentada a un proceso regional que responde, sin lugar a dudas, a los modelos conceptuales y sistemas institucionales norteamericanos; cuya difusión contaba con impulsos muy precisos por parte del gobierno de EE. UU. y organismos internacionales (ver Otero y Selis, 2016). Pero, si bien los procesos de escala global, regional y nacionales más amplios influyeron sobre las características del programa de formación estudiado, éste implicó también una perspectiva que incorporaba una región específica (La Plata y otros nueve partidos circundantes) como área de intervención de una Universidad Nacional. El programa traía al aula la experiencia de técnicos de las agencias de extensión de otra institución del complejo científico-tecnológico nacional (INTA) y proponía que los estudiantes avanzados de una carrera de grado lleven a cabo prácticas en terreno.

Sintéticamente, decimos que la enseñanza universitaria de la extensión agropecuaria tanto a nivel de grado como de posgrado ${ }^{3}$ fue institucionalizada en un período inaugurado por violentos gobiernos de facto que a su vez se proponían proyectos modernizantes y que es recordado por grandes movilizaciones y luchas del movimiento obrero y estudiantil argentino.

Dicho el contexto, consideramos que el proceso de determinación curricular de la disciplina extensión agropecuaria se vuelve cautivante y pertinente para aportar a los debates teóricoconceptuales y metodológicos con relación al campo actual del conocimiento de la extensión universitaria. Nuestra meta es que esta reconstrucción supere lo disciplinar y abone a una reflexión más amplia sobre la importancia de la formación teóricometodológica en "extensión crítica" (Tommasino y Hegedus, 2006), la "curricularización de la extensión", la "integralidad de funciones" (Arocena, 2010; Tommasino y Rodríguez, 2010); la "educación experiencial" (Menéndez y otros, 2013), entre otras discusiones vigentes.

\section{Apunte breve sobre la determinación curricular}

Un breve apunte sobre el concepto de curriculum sirve para introducir la idea de que no es posible pensar la introducción de la extensión agropecuaria en la currícula de grado como un proceso lineal y mecánico. Antes que sólo presentar cómo estaría reflejando la imposición lineal de un modelo foráneo (el estadounidense) en el ámbito local, nos permitimos pensarlo como un proceso más complejo. En este sentido, Alicia De Alba indica que el curriculum reúne:

"elementos culturales (conocimientos, valores, costumbres, creencias, hábitos) que conforman una propuesta políticoeducativa pensada e impulsada por diversos grupos y sectores sociales cuyos intereses son diversos y contradictorios" (De Alba, 1998:76).

La determinación curricular es el proceso de génesis de esa síntesis que deviene de un juego de negociaciones e imposiciones sociales propio de determinado momento histórico. De esto participan sujetos sociales con interés en (y poder para) fijar su orientación y sus rasgos centrales. Del mismo modo, el proceso de estructuración formal del curriculum y el desarrollo curricular tienen sus propios agentes: por un lado, aquellos que le dan forma en el ámbito institucional y, por otro lado, aquellos que lo convierten en una práctica cotidiana. Retomando a Bourdieu (1970), la autora citada afirma que hay que entender al curriculum como un arbitrario cultural, antes que como un sistema congruente y articulado. Sin dudas, no podremos dar cuenta en toda su complejidad de los procesos de determinación, estructuración y desarrollo curricular asociados al origen a la Cátedra de Extensión Agropecuaria en la UNLP en 1969. Sin embargo, presentamos una reconstrucción basada en la revisión bibliográfica y en los resultados de análisis documental haciendo hincapié en dos cuestiones: a) un recorrido histórico por las conceptualizaciones y las instituciones de la extensión agraria entre fines del siglo XIX y los años 60 y b) las actividades de docencia/investigación/extensión implícitas en el Programa de Capacitación en Extensión Agropecuaria iniciado en 1966-1967.
2) "Argentina 1966 supuso una transferencia en la distribución de la plusvalía en perjuicio de la burguesía pequeña y mediana y de la llamada "oligarquía agropecuaria", proceso al que se superpuso un flujo constante de ingresos en favor del Litoral en detrimento del
Interior. Una política de tal modo agresiva, que busca quebrar una situación de "empate", no puede desatarse sino a través del respaldo de la violencia desnuda, montada sobre una estructura vertical, autoritaria del Estado" (Portantiero, 1973:13, en Cirio, 2014:104).
3) Nos remitimos a la formación dentro del ámbito universitario, pero incluso la Escuela para Graduados en Ciencias Agropecuarias ubicada en Castelar es una entidad establecida por convenio IICA, INTA, UNLP y Universidad Nacional de Buenos Aires en junio de 1967 (Documentos del Posgrado de Formación en Extensión, Escuela para Graduados en Ciencias Agropecuarias 1972). 


\section{Una periodización ad hoc: del fomento agrario a la extensión agropecuaria}

En el recorrido histórico "del fomento agrario a la extensión agropecuaria" en Argentina se halla no sólo un panorama de las perspectivas, desafíos e intervenciones relacionadas a esta temática, sino que también aparecen mencionados los antecedentes institucionales directos de la Facultad de Agronomía de La Plata.

\subsection{El fomento agrario}

En Argentina, podemos hallar las primeras experiencias de extensión agropecuaria -en un sentido amplio y abarcativo del término- con fines educativos $y / o$ productivos, entre fines del siglo XIX y principios de siglo XX. En esa época distintas esferas estatales comienzan a asumir medidas dirigidas, en última instancia, a mejorar indicadores productivos (volumen, calidad) fomentando el uso de determinadas técnicas y/o insumos así como a extender la frontera agrícola. Una de las medidas más importantes fue la promoción a rango de Ministerio en el año 1898 del Departamento de Agricultura (que había sido creado en 1871, dependiente del Ministerio del Interior), el cual tenía entre sus propósitos tanto la formación de técnicos como la difusión de información que promuevan el incremento de la productividad de la tierra. Djenderedjian indica que:

"De ese modo, uno de los medios fundamentales de transformación que comenzó a ser visto como una necesidad fue la creación de un instrumento estatal con la envergadura y la capacidad suficientes como para cambiar la realidad agraria a través de la coordinación sistemática de organismos y programas de fomento agrario, y capaz asimismo de realizar en gran escala la experimentación y difusión de nuevos métodos agrícolas". (2013:14)

Las acciones iniciales iban destinadas a incorporar nuevos cultivos, fomentar el uso de semillas mejoradas y aplicar técnicas que permitan incrementar rendimientos.

El autor citado afirma que, en la misma época, además del Ministerio, hubo otras novedades institucionales de procedencia tanto estatal como privada, orientadas a desarrollar la formación, la experimentación y la literatura especializadas ${ }^{4}$ en torno a la actividad agropecuaria. Indica que la generación, recopilación y difusión de información técnica y estadística, así como las tareas de difusión de conocimientos y técnicas mejoradas de cultivo y cría de ganado figuraban en el presupuesto del Ministerio, mientras que las escuelas agropecuarias —cuyo objetivo fundamental era el de formar prácticamente a sus alumnos en las técnicas relativas a la producción- fueron una de las instancias de formación en producción agropecuaria. Asimismo, a fines del siglo XIX fueron creadas las primeras instituciones dedicadas exclusivamente a la formación práctica y "científica" en agronomía y veterinaria: la Escuela Agronómica de Mendoza y el Instituto AgronómicoVeterinario de Santa Catalina, en Lavallol, hoy partido de Lomas de Zamora en el conurbano bonaerense. Este último, tiene como antecedente la Escuela Práctica de Agricultura de Santa Catalina, creada en 1872. El Instituto Agronómico-Veterinario de Santa Catalina es el antecedente histórico directo de la actual Facultad de Ciencias Agrarias y Forestales, que en el período que abarca este artículo, se denominaba Facultad de Agronomía de La Plata. ${ }^{5}$ Aquello que contemporáneamente podemos incluir dentro de una noción de extensión y que se contemplaba con el nombre de fomento agrario, tenía a la estación experimental agrícola como núcleo de experimentación y difusión, muy al estilo consolidado de los Land Grant College norteamericanos, creados por la Ley Federal conocida como "Smith-Lever Act" en 1914 (Otero y Selis, 2016). Este modelo se perfecciona y se generaliza en todo el territorio nacional, fundamentalmente en áreas extrapampeanas (Gargano, 2011). A principios del nuevo siglo, se crean el Servicio de Agronomías Regionales ${ }^{6}$ y la Oficina de Estaciones Experimentales, dependientes del Ministerio de Agricultura y Ganadería de la Nación. Cabe resaltar que a cargo de la primera Agronomía Regional estuvo el italiano Hugo Miatello quien es considerado "pionero de la educación y extensión rural en Argentina" (Tranchini, 2013:381).

Para 1920 el sistema funcionaba en red, compartiendo información y resultados de ensayos y experimentaciones. Alemany (2012) señala que mantiene su formato hasta principios de la década del '40. En 1944 se da un nuevo paso al crearse la Dirección de
4) El autor señala: “La Quinta de Aclimatación de Santa Cruz, creada en 1885 , contó desde 1893 con un agrónomo especializado; las colonias oficiales formadas por impulso nacional ( $y$ a veces también administradas por el Estado) en diferentes provincias y territorios fueron en algunos casos acompañadas por escuelas de agronomía (por ejemplo, la de Caroya, en Córdoba) o campos de ensayos (la de Yeruá en Entre Ríos); proyectos de nuevas escuelas agronómicas fueron llevados a cabo también en Bella Vista, en Corrientes, en 1894, o en Córdoba, en un plan para crear cuatro de ellas sancionado en el presupuesto de 1895. La literatura producida por las diversas instancias estatales y privadas crecía sin cesar." (Djenderedjian, 2013: 15) 5) El Instituto Agronómico Veterinario de Santa Catalina deviene Facultad de
Agronomía y Veterinaria de la provincia de Buenos Aires en 1889 y ésta es trasladada a la ciudad de La Plata en 1890. En 1905 esta institución pasa a formar parte de la flamante Universidad Nacional de La Plata (UNLP). En 1921 Agronomía y Veterinaria se establecen como unidades académicas autónomas. En los años 60 es creada la Escuela Superior de Bosques para la enseñanza de la carrera de ingeniería forestal. Finalmente, la UNLP introduce en 1990 el actual nombre de su precedente Facultad de Agronomía y ésta pasa a llamarse Facultad de Ciencias Agrarias y Forestales.

6) La primera Agronomía Regional fue creada en la localidad bonaerense de Mercedes en abril de 1908. Promediando el siglo XX, las Agronomías Regionales pasarán a ser Agencias de Extensión Rural del flamante INTA. 


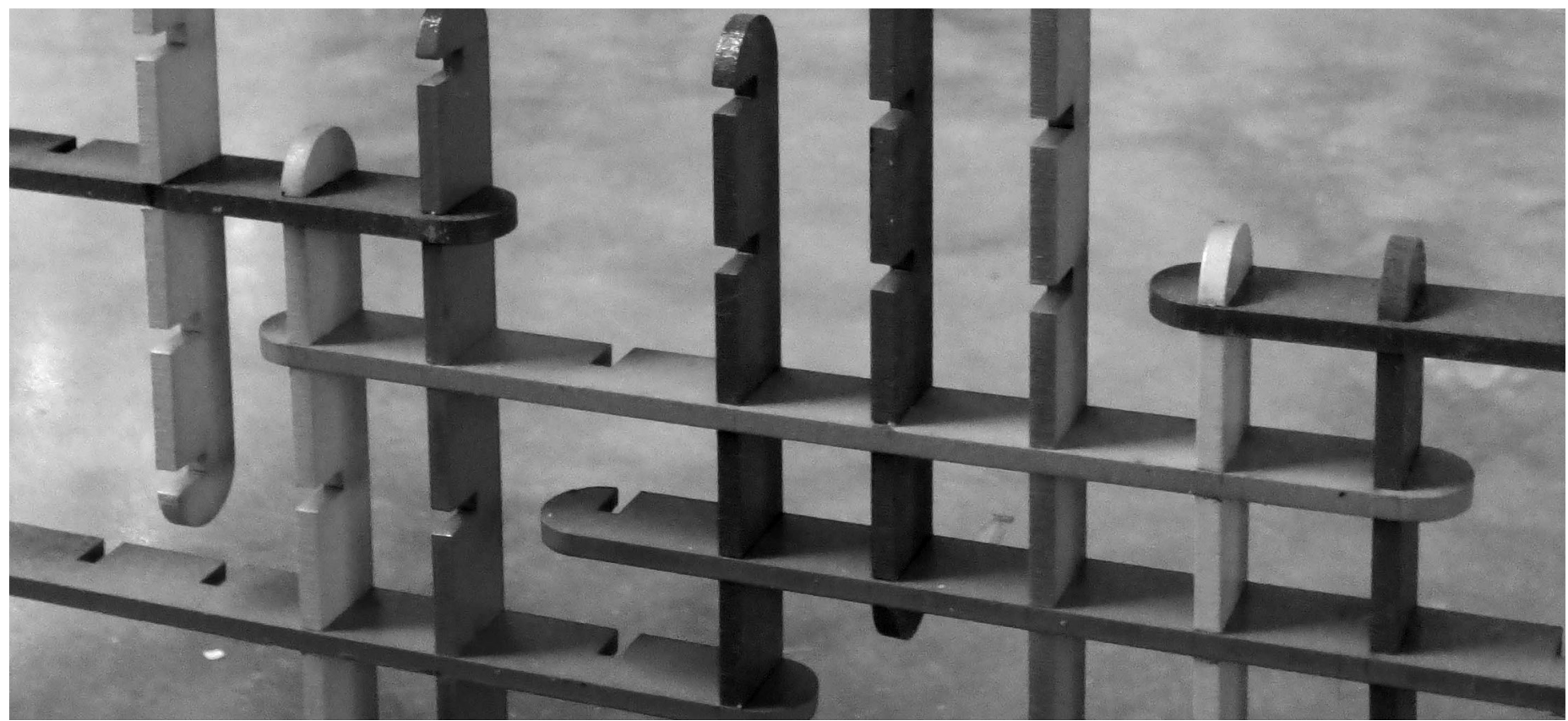

(C) Micaela Block

Enseñanza Extensiva, dentro de la Dirección General de Enseñanza Agrícola, como instancia de difusión de técnicas y tecnología agropecuaria, dentro del Ministerio de Agricultura y Ganadería. Asimismo, se crean Clubes Agrícolas dedicados a la juventud rural, antecedente de los Clubes $4 \mathrm{~A},{ }^{7}$ mientras que la mirada sobre la mujer en el espacio rural se orienta hacia la economía doméstica (Gargano, 2011). Se dirige la mirada hacia la familia rural, pero desagregándola según un modelo teñido de raíces religiosas. En términos de cómo se entendía la extensión, la divulgación de información generada en las distintas instancias estatales y privadas era la estrategia para mejorar la productividad de la tierra: las mejoras se suponían - y en muchos casos se alcanzabanproducto de la difusión de técnicas, del uso de material informativo y materiales genéticos mejorados y el trabajo junto a productores de profesionales sin una formación específica para la intervención en el medio, pero dotados de materiales e información técnica necesaria para superar la "brecha tecnológica". No encontramos en la bibliografía de la época referencias a una formación particular de los técnicos en una metodología que facilitara la transmisión de la información generada en los centros de investigación y adaptación. Es un difusionismo que concebía al estímulo de factores exógenos como inductor de la modernización agraria. Por un lado, de acuerdo con los enfoques teóricos que Bravo (2001) identifica en un trabajo sobre distintas perspectivas del fenómeno de la innovación en el medio rural, este es un período en el que la innovación tecnológica se entendía como de innovación inducida por factores del entorno macroeconómico y por el propio "estado de la ciencia" que dotaba de ciertas cualidades al objeto técnico. Este período se prolonga hasta los años 50 . Por otro lado, si nos remitimos a los modelos de comunicación la extensión (aunque es más pertinente hablar de fomento agrario) se definía bajo un modelo exógeno con énfasis en la información (ver Kaplún, 1985). Alcanzaba con una formación técnica para transferir las novedades tecnológicas. En este sentido, se puede decir que hasta mediados del siglo $X X$ la extensión agropecuaria era una intervención sin formación, o bien, que alcanzaba con haber recibido instrucción (tanto sea universitaria o terciaria) en disciplinas vinculadas con las ciencias agropecuarias para promover mejoras en la producción. Esto se verá modificado a partir de la década del ' 50 , cuando comienza una nueva etapa para la extensión en estas latitudes — que superará de alguna manera la noción de fomento agrario- para dar lugar a un abordaje disciplinar, sistemático y metódico, con el fin de influir en la conducta del productor agropecuario y su familia. La extensión dejará de ser "fomento" para ser "educación". 


\subsection{La etapa de la formación en la posguerra}

Finalizada la Segunda Guerra Mundial, una serie de hechos moldeó un cambio en la mirada sobre lo rural, la producción y el productor en toda América Latina: EE. UU. buscaba consolidar su hegemonía sobre el continente, territorio con una población rural más que significativa y gran productora de materias primas, fundamentalmente alimentos. Al mismo tiempo, en el marco de la Guerra Fría, hechos políticos como la revolución cubana y la presencia de movimientos revolucionarios - con fuerte componente campesino- suponían un riesgo para el lugar que EE. UU. le asignaba a América Latina, como proveedora de alimentos a su fortalecida industria alimenticia. En este sentido, el concepto de agribusiness viene a promover —o bien sintetizar- el desarrollo de una agroindustria dominante y demandante de grandes volúmenes de materias primas para lo cual había que modernizar un campo "atrasado" y generar productos agroalimentarios en grandes volúmenes y estandarizados, a la vez que promover una relación fuertemente dependiente de los productores con la gran industria. En este contexto se fue consolidando una mirada sobre la población objetivo de la intervención: la familia rural. En ese entonces, el escenario de las propuestas de modernización era definido como un campo latinoamericano dual, en el cual la presencia del latifundismo improductivo convive con el minifundio ineficiente. Este agro atrasado, según el enfoque dominante entre los expertos norteamericanos, tenía que transformarse. Y aquí se cruzan la propuesta desarrollista, que busca, a través de la modernización liberar mano de obra campesina para la industria urbana —sustitución de importaciones mediante-y las necesidades de la gran industria alimentaria. De esta forma comienzan a conjugarse la mirada norteamericana de la extensión, sostenida en enfoques de la sociología y la comunicación propios de los modelos del consenso (o del equilibrio o del orden, según Sánchez de Puerta, 1996) con el diagnóstico cepalino. ${ }^{8}$ Acorde con esta tendencia modernizante en América Latina, comienza a pensarse la formación en extensión agropecuaria como necesaria para acelerar tiempos y resultados. Las propuestas de formación se complementan con publicaciones que responden directamente al modelo norteamericano en su proyecto continental, como la revista Extensión en las Américas. En la década del 50 son introducidos en la región los primeros cursos y se crean organismos de carácter nacional especializados en desarrollo agrotecnológico. El Instituto Interamericano de Cooperación para la Agricultura (IICA) perteneciente a la Organización de los Estados Americanos (OEA) con sede en Costa Rica, será clave para este despliegue.
Entre los hitos de la década del '50 podemos destacar tres: 1) El dictado del "Primer curso de Extensión para América Latina" impulsado por el IICA-OEA y la Organización de las Naciones Unidas para la Alimentación y la Agricultura —conocida mundialmente como FAO- en 1952 en Montevideo. En dicho curso participaron técnicos de la región, quienes posteriormente serán multiplicadores de los conceptos trabajados.

2) En 1955, un seminario de extensión en la Estación Experimental de Pergamino — preexistente a la creación del INTA — dictado por técnicos de la OEA, recibió a cuarenta agrónomos regionales (Alemany, 2012). Por esta época, las estadías de los profesionales en el exterior y las capacitaciones que seguían un modelo definido fronteras afuera completaban el cuadro de la formación de los técnicos en extensión (Gargano, 2011; Barrientos, 2002).

3) La creación del Instituto Nacional de Tecnología Agropecuaria en el 1956, siguiendo las políticas continentales a favor de la institucionalización de la modernización del campo. Su creación marca un hito en la intervención del Estado en la investigación y difusión de mejoras en la producción agropecuaria, al tiempo que responde a la concepción de la extensión que se estaba generalizando en el continente, así como a la necesidad de formar técnicos para llevar a cabo la tan requerida modernización, al menos hasta fines de los ' 60 .

En 1960 se dicta el "Segundo Curso Internacional de Economía Doméstica en Extensión Agrícola", realizado en Concepción del Uruguay y organizado por el IICA, que reafirma estos preceptos y el lugar que le cabe al productor y a la familia rural (ver Gutierrez, 2009). Según Alemany (2009), en esta primera etapa, en el INTA predomina el modelo educativo, caracterizado por promover la modernización del agro local, involucrando a la familia —aunque siempre atribuyéndole roles y lugares diferenciales al hombre, a la mujer y a la juventud-. Siguiendo a Kaplún (1985), entendemos que la concepción que se tenía de la comunicación se ubicaba en un modelo que buscaba la modernización deseada a través de la persuasión de la población rural. Y la formación de los técnicos, ahora sí, iba en esa dirección.

\section{La extensión camino a la currícula de grado}

\subsection{EI "Expediente X": colaboración INTA y FALP 1966-1975} En la introducción decíamos cuán significativo fue para nosotros sumergirnos en las más de 130 fojas del Expediente
8) El pensamiento de la Comisión Económica para América Latina y el Caribe (CEPAL), que Naciones Unidas activa en 1948 y donde se desarrolló una versión regional de las teorías de desarrollo. Por esta época, el énfasis del pensamiento cepalino estaba en la industrialización de la región. Ver Bielschowsky, 1998 
200-24194/67, referido a la colaboración entre la FALP y el INTA durante 1966 y 1975 para el desarrollo de un Programa de Capacitación en Extensión Agropecuaria en el ámbito de la FALP. Del contenido explícito del convenio se desprenden no sólo la presencia de determinado paradigma teórico-metodológico sino también cuestiones logísticas y de coyuntura que atravesaban el desarrollo del Programa.

En este documento es posible encontrar diversos actores entrelazados en distintos niveles. Aproximadamente 50 personas son mencionadas, cuya pertenencia institucional se circunscribe mayoritariamente a FALP (con cargos de decanos, vicedecanos, interventores, secretarios, jefes de departamento, docentes del Programa en cuestión y otros) e INTA (presidentes, interventores, directores, técnicos de Agencias cercanas, docentes del Programa en cuestión y otros). En menor medida aparecen funcionarios de organismos de gobierno de la provincia de Buenos Aires (como Ministerio de Asuntos Agrarios e Inspección General de Escuelas, entre otros), mientras que sólo un empresario de la región es mencionado: el gerente de los frigoríficos Armour y Swift, a quien se solicita que responda una encuesta.

Según lo expresado en el expediente, el Programa comprendió inicialmente cinco actividades: dos cursos de grado programados para 1967 y 1968; un Estudio de Situación de la "zona de influencia" de la Facultad de Agronomía; la capacitación del plantel docente y la no menos importante tarea de organización de los elementos didácticos (preparación de los programas, selección de la bibliografía, materiales, etcétera).

En primer lugar, con respecto a los cursos, el curso de Orientación era obligatorio y duraba dos meses. Estaba destinado a estudiantes de $4^{\circ}$ año de Agronomía, a los que se pretendía dar conocimientos básicos sobre el proceso de extensión y la actuación profesional del extensionista. Al mismo tiempo, el desarrollo incluía instancias de panel en el que intervenían extensionistas de las Agencias de Extensión INTA circundantes: Coronel Brandsen, Pereyra Iraola y Magdalena. El curso de Formación, por su parte, era optativo y estaba dirigido a alumnos de $5^{\circ}$ año. La carga teórico-práctica duraba entre marzo y noviembre, a las que se sumaban horas de "intensificación de prácticas" en las áreas de influencia de las Agencias mencionadas.

En segundo lugar, acerca del plantel del Programa de Capacitación en Extensión Agropecuaria en este periodo se destacan dos figuras: por un lado, el ingeniero agrónomo Rolando Jacinto Vellani, quien cumplió actividades docentes primero como colaborador (por INTA) y, a partir de 1970, con el cargo de Profesor Titular Libre de la flamante cátedra. Por otra parte, el ingeniero agrónomo Isvert Valdemar Cometta, técnico de la Dirección de Colonización del Ministerio de Asuntos Agrarios de la Provincia de Buenos Aires, quien fue el coordinador del Programa por parte de la FALP. Desde 1970 ocupó el cargo de Profesor Adjunto de la cátedra. Son ellos los interlocutores clave, quienes remiten o son remitentes de las comunicaciones (notas, informes, proyectos, etc.) incluidas en el expediente del Convenio.

En tercer lugar, el "Estudio de Situación" pretendía describir el área geográfica constituida por los partidos de La Plata, Berazategui, Coronel Brandsen, Ensenada, Florencio Varela, General Paz, Quilmes, Berisso y San Vicente. El estudio comprendía cuatro etapas, una de descripción de todas las localidades de la región en aspectos económico-productivos, socio-demográficos e históricos. Las etapas siguientes pretendían determinar y caracterizar en el área de trabajo los "sectores de producción homogéneos", "la organización y manejo técnico-económico de los predios agrícolas" y por último los "factores sociales, culturales y económicos asociados con la adopción de prácticas agropecuarias" (foja $\mathrm{n}^{\circ} 81$, la cursiva es nuestra).

Por último, en cuanto a los programas y la bibliografía seleccionada es notable observar las líneas de continuidad con el modelo de Extensión Rural que EE. UU. había impulsado a través del IICA. La sociología rural norteamericana, la antropología cultural y la psicología educativa conductista ostentaban un lugar privilegiado en los programas y manuales de lectura compilados por Vellani para el desarrollo del Programa. Tanto es así que las publicaciones de la revista Extensión en Las Américas son parte mayoritaria de la selección bibliográfica y Joseph Di Franco, uno de sus emisores privilegiados, el autor más citado ${ }^{9}$ del manual de lectura; junto con Luis Carlos Cruz y George McClelland Foster. ${ }^{10}$

La pretensión de contribuir no sólo a la formación de "ingenieros agrónomos con orientación en extensión agropecuaria" sino de aportar en términos teórico-metodológicos a la pregunta por la adopción tecnológica es explícita en el expediente. En este sentido, hay un posicionamiento teórico claro, pero a su vez, la promesa de que desde estas latitudes podía aportarse algo original, debido justamente, a su especificidad y que ello posibilitaría además "un reajuste de los planes de la Facultad a los problemas reales de los productores". (foja $n^{\circ} 1$ bis)
9) Otero y Selis (2016:10) afirman que: "En los números analizados, los autores que aparecen con mayor frecuencia presentando artículos con contenido conceptual sobre la extensión rural son Joseph Di Franco y Earl Jones.
(...) Joseph Di Franco escribe sobre la 'esencia' de la extensión y el desarrollo de la comunidad. Fue un norteamericano de origen italiano. Se educó en las instituciones del Estado de Ohio, donde también trabajó en el Servicio de Extensión. Realizó el doctorado en Educación en la Universidad de Columbia, y luego trabajó como Profesor en la Universidad de Cornell. En 1958 ingresó al IICA y ocupó cargos de alta jerarquía como 'Jefe de la Disciplina de
Economía y Ciencias Sociales'." 10) Este último es autor de un trabajo antropológico titulado "Las culturas tradicionales y los cambios técnicos" (1964). 


\section{Conclusiones}

La perspectiva que desarrollamos a lo largo de estas páginas fue analizar el contexto histórico, los espacios institucionales, los enfoques y otros elementos de un proceso de determinación curricular para la formación de extensionistas ocurrido en La Plata en las década del '60.

Entendimos que ya a fines del siglo XIX-principios del siglo XX, con la incipiente pero compleja institucionalización del "fomento agrario" como cuestión de Estado, fue que irrumpió - de manera embrionaria- un problema que consideramos clave al hablar de formación de extensionistas y que podríamos sintetizar como el problema de la producción de cierto tipo de información y de cierto tipo de agentes capaces de encarnar objetivos de transformación de la realidad (agraria, en este caso); coordinados por instancias (estatales y/o privadas) más amplias. Este problema clave obtuvo un tipo de respuesta en el marco conceptual y en el sistema institucional de extensión configurados en los años '50, con un "modelo educativo" de clara influencia norteamericana. Ésta se comprueba en el programa de formación en extensión agropecuaria estudiado, lo cual guarda relación con procesos globales ocurridos luego de la Segunda Guerra Mundial particularmente en el discurso y en la implementación de políticas orientadas por el binomio desarrollo/subdesarrollo.

Interpretamos finalmente que, en consonancia con una vocación "práctica" de determinación de un espacio político y pedagógico específico, el Convenio INTA-UNLP del que surge la Cátedra de Extensión Agropecuaria en el '69 platense constituyó también un paso del "territorio a la currícula". Introdujo, desde un enfoque difusionista y con huellas visibles de las teorías de la modernización, actividades de investigación (el Estudio de Situación) y docencia; dispositivos que acercaban a los estudiantes a las problemáticas específicas del desarrollo de la región (La Plata y otros partidos circundantes) y a las prácticas profesionales extensionistas.

Reconstruyendo los antecedentes y la génesis de un proceso ocurrido hace 50 años en la Universidad pública, pretendimos poner sobre la mesa algunas tensiones (y posibilidades) vigentes no sólo en el campo de la extensión rural, sino también en el de la extensión universitaria.

Las tensiones aparecen en los planteos dialógicos actuales de la formación en extensión rural —-producto de una revisión de los contenidos y de una mirada crítica del sentido que tiene la intervención en el medio-y la tradición con sesgo difusionista que impera en dichas intervenciones. En la coexistencia y la tensión entre estos mismos enfoques en los sistemas de extensión universitaria, tanto como en los vínculos entre lo local, lo nacional y lo global de lo que se vuelve materia de debate en determinado momento histórico; se encuentra la complejidad y parte de las contradicciones que deberán ser disputadas si se iniciaran o profundizaran procesos curriculares para la formación de extensionistas en otros campos.

Las posibilidades residen en la potencia de aquella promesa ${ }^{11}$ de hacer aportes originales desde la especificidad local y de aquel objetivo que en las primeras fojas del convenio descripto llamaban "reajuste de los planes" a partir de la vinculación con otros actores y sus problemáticas. En el presente, intentando superar la dicotomía intramuros/extramuros, estos aspectos nos remiten a la búsqueda de transformaciones no sólo en el difuso "allá afuera". Desde una óptica reflexiva y crítica de un proceso estudiado en el difuso "acá adentro" (o de una moneda que se derrite y cuyas caras se vuelven más difíciles de apreciar con claridad) afirmamos que es esto lo que rebasa el campo de la extensión rural y constituye nuestro puente con los debates de la extensión universitaria.

Por último, queda abierto el desafío de superar la discusión entre extensionistas. Otros actores universitarios y no universitarios se hallan entrelazados cotidianamente por y con estos discursos y prácticas sociales. Involucrarlos en las discusiones y acciones dirigidas hacia propuestas político-educativas de formación en extensión sería una forma de reconocer y trabajar sobre la base de la compleja "arbitrariedad cultural" del curriculum. Enriquecer esta discusión (negociar/confrontar sus múltiples significados) no se trata de un afán voluntarista y participativo en sus formas. Se trata de una búsqueda colectiva de salidas a los modelos lineales y unidimensionales, sin perder de vista el reto de interpelar en lo concreto a los responsables de la extensión.
11) Hemos elegido la palabra "promesa" aunque no asignamos per se una connotación positiva o negativa a lo que decimos que constituye. Cabe resaltar que las propuestas y posturas ideológicas detrás de un mismo objetivo aparente pueden ser variadas y hasta contrapuestas. Eso es parte de lo que es disputado y negociado en este tipo de procesos. 


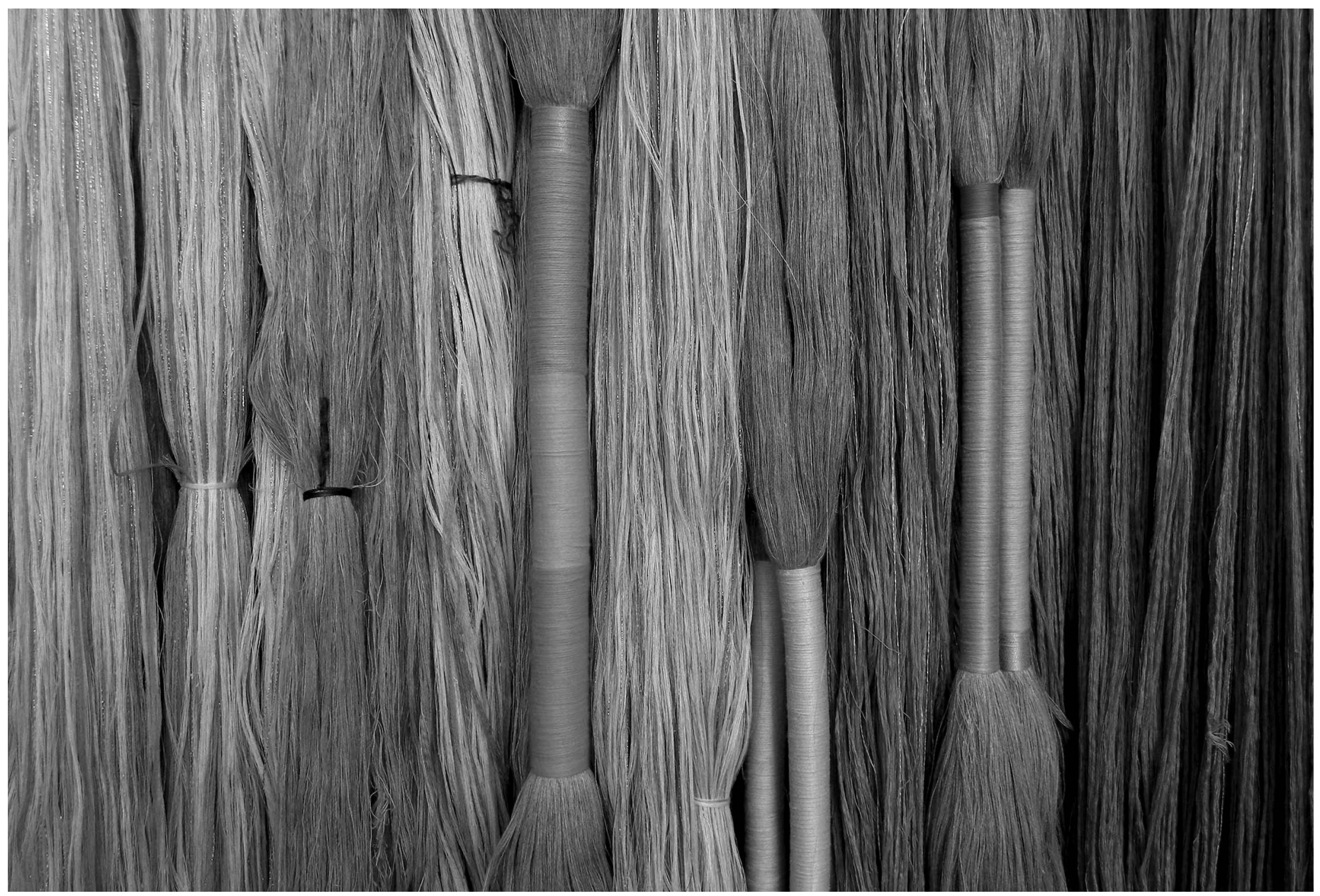

() Jorge Malachesky

\section{6 \\ la pretensión de contribuir no sólo a la formación de "ingenieros agrónomos con orientación en extensión agropecuaria" sino de aportar en términos teórico- metodológicos a la pregunta por la adopción tecnológica es explícita}




\section{Referencias bibliográficas}

Alemany, C. (2009). Los cambios de la extensión del INTA y su relación con los paradigmas del desarrollo. Buenos Aires: INTA

Alemany, C. (2012). Elementos para el estudio de la dinámica y evolución histórica de la extensión rural en Argentina. Tesis doctoral. Instituto de Sociología y Estudios Campesinos (ISEC) Universidad de Córdoba. Córdoba, España: Servicio de Publicaciones de la Universidad de Córdoba.

Arocena, R. (2010). Curricularización de la Extensión: ¿porque, cuál, cómo? Integralidad: tensiones y perspectivas. Cuadernos de Extensión, (1), 9-18.

Barrientos, M. (2002). Evolución de los servicios de extensión en nuestro país. Vinculación con los planteos pedagógicos vigentes. Compendio Bibliográfico de Extensión Rural. Córdoba: FCA-UNC.

Bielschowsky. R. (1998). Evolución de la ideas de la CEPAL. Revista de la CEPAL, número extraordinario (octubre), 21-45. Santiago de Chile.

Bourdieu, P. y Passeron, J.C. (1970). La réproduction. Paris: Minuit.

Bravo, G. (2001). Procesos de innovación agropecuaria: un punto de vista sobre sus características en una perspectiva de gestión. II Jornadas Interdisciplinarias en Estudios Agrarios y Agroindustriales. Facultad de Ciencias Económicas, UBA, Buenos Aires, 6-8 de noviembre.

Campos, L. (2010). La segunda fase de la sustitución de importaciones. En Socolovsky, Y. y Arceo, N. (Comps.), Desarrollo económico, clase trabajadora y luchas sociales en la Argentina contemporánea. IEC-CONADU.

Cavarozzi, M. (1983). Autoritarismo y democracia 1955-1983. Buenos Aires: Centro Editor de América Latina.

Cirio, G. (2014). Planificación territorial y acumulación capitalista. Vínculos entre modelos de acumulación y políticas de planificación del territorio argentino 1955-2014. Realidad Económica (286), 93-121.

De Alba, A. (1998). Curriculum: crisis, mito y perspectivas. Buenos Aires: Miño y Dávila.
Djenderedjian, J. (2013). El peso útil del estado. Las políticas gubernamentales de desarrollo tecnológico, investigación y extensión rural en la Argentina de inicios del siglo XX. Historia Política, 12, 1-24.

Gargano, C. (2011). Rupturas y continuidades en investigación y extensión rural: el Instituto Nacional de Tecnología Agropecuaria (INTA) durante la última dictadura cívico-militar argentina (1976-1983). XIII Jornadas Interescuelas. Departamentos de Historia Catamarca.

Gutierrez, T. (2009). Agro pampeano y roles familiares en la década de 1960. Mundo Agrario, 10 (19) (Segundo semestre).

Kaplún, M. (1985). El comunicador popular. Parte I. Quito: Ciespal.

Menéndez, G. y otros (2013). Integración docencia y extensión. Otra forma de enseñar y de aprender. Santa Fe: Ediciones UNL.

O’Donnell, G. (1977). Estado y alianzas en la Argentina, 1956-1976. Desarrollo Económico, 16 (64, enero-marzo), 523-554.

Otero, J. y Selis, D. (2016). La revista "Extensión en las Américas". Influencia de los EU. UU. en los servicios de extensión rural latinoamericanos. Extensão Rural, 23 (1). Recuperado de: http://cascavel.ufsm.br/revistas/ojs-2.2.2/index.php/extensaorural/article/view/17359 (consultado el 20 de marzo de 2016).

Sánchez de Puerta Trujillo, F. (1996). Extensión agraria y desarrollo rural. Sobre la evolución de las teorías y praxis extensionistas. Madrid: Ministerio de Agricultura, Pesca y Alimentación. Serie Estudios nº 123.

Tommasino, H y De Hegedüs, P. (2006). Extensión: reflexiones para la intervención en el medio urbano y rural. Montevideo: UDELAR - Facultad de Agronomía. Tommasino, H y Rodríguez, N. (2010). Tres tesis básicas sobre extensión y prácticas integrales en la Universidad de la República. Cuadernos de Extensión, (1), 19-42.

Tranchini, E. (2013). Granja y Arado. Spenglerianos y Fascistas en la Pampa 1910-1940. Buenos Aires: Dunken.

\section{Documentos}

Vellani, R. J. (1970). Manual de lectura. Cátedra de Extensión Agropecuaria. Facultad de Agronomía, Universidad Nacional de La Plata. 226 pp.

Vellani, R. J. (1967). Temas desarrollados en las clases del primer curso de orientación en extensión agropecuaria. Programa de Capacitación en Extensión Agropecuaria. Facultad de Agronomía, Universidad Nacional de La Plata. 43 pp. Convenio con el Instituto Nacional de Tecnología Agropecuaria (INTA), de un trabajo coordinado para el desarrollo de un programa de extensión agropecuaria Archivo de la Mesa de Entradas de la Facultad de Ciencias Agrarias y Forestales, Universidad Nacional de La Plata. 1967. Expediente 200-24194/67.

Naumann y Vellani (1972). Seminario de Desarrollo Curricular de Extensión Agropecuaria. Escuela para Graduados en Ciencias Agropecuarias. Castelar. 22-24 de mayo.

Programa de Posgrado de Formación en Extensión. Escuela para Graduados en Ciencias Agropecuarias 1972.

Nota al Secretario de la FALP firmada por Valdemar Cometta, 22 de noviembre de 1967

Programas de los Cursos de Orientación y Formación en Extensión Agropecuaria. Programa de Capacitación en Extensión Agropecuaria. Facultad de Agronomía, Universidad Nacional de La Plata, s/d. 